\title{
Telenursing as a new nursing paradigm in the 21 century: A literature review
}

\author{
1.2. Al Afik, ${ }^{3 .}$ Moses Glorino Rumambo Pandin Dr. M.Si., M.Phil., M.Psi., Psikolog \\ 1. Program Doktor Nursing Faculty Universitas Airlangga Surabaya \\ 2.Nursing Program Medical and Health Science Univesitas Muhammadiyah Yogyakarta \\ 3. Department of English Literature, Faculty of Humanities \\ , Universitas Airlangga
}

Corespoden Author : Al Afik

Email : al.afik-2020@fkp.unair.ac.id

\begin{abstract}
Nursing is a part of health services that serves individuals, groups and communities. In this 21 st century the need for nursing services has changed rapidly and in a complex manner. Physical limitations, is on should to be considered, while the implementation and maintenance of patient-centered service infrastructure still have to be recked. The aim of this literature review is to identify the role of telenursing in nursing services for patients. This language method uses literature reviews which are summaries of 10 articles in the publication years of 2019, 2020, and 2021 on 3 search databases, namely Scopus, Science Direct, and SAGE. This review used the new Joanna Briggs Institute and Prisma. The eligibility of these studies were from its title, abstract, research methodology, results, and discussion. The results of the review were presented in narrative form. The results of a review of 10 articles found that the form of nursing services with telenursing could prove long-distance service, time efficiency and funding allocation, but in terms of quality it had been not satisfactory related to its physical implementation of nursing service. Conclusion: The nursing paradigm in the 21 st century global era has been developed with the help of technology to meet the needs of distance efficiency and cost limitations. Thus, telenursing is a solution to answer these challenges.
\end{abstract}

Keyword: Telenursing- Nursing- 21st century paradigm

\section{INTRODUCTION}

The nursing paradigm in the 21 st century is interpreted not only as a perspective and thought process, but requires a change in strategy in all matters related to efforts to provide holistic nursing care including bio-psycho-socio-spiritual to individuals, groups and society. Nurses as part of change agents have a share in various fields, including the use of appropriate technology for the nursing care process. Nevertheles, it has been limited due to the physical condition, burden, location of the patient, and geographic location-while the current instructions for the implementation and maintenance of health must be patient-centered(1).

In this 21 st century, the need for health services continues to experience rapid changes. Health care needs are increasingly complex with various health problems. This encourages an increasing need to create new and innovative health service models in accordance with the demands of the times (2). The use of technology is a very appropriate strategy and is needed to meet the needs of care in a sustainable manner. Technology is a means for health services to be able to increase the response to care needed by the community. This provides a new window for improving care services (3). Subsequently, advances in technology are the main force to 
overcome various kinds of health problems that are currently occurring - in order to provide health services more widely and can be accessed more easily for those who need it (4).

Telenursing is a technology-based nursing service that was created to provide convenience to health services for patients (5). The existence of telenursing for health services is based on many things, such as: the availability of limited human resources which makes it is unable to reach more patients, the limited capacity of hospitals and infrastructure, the need to minimize the risk of exposure to infection between patients and health workers, and the access to health services (4).

The existence of telenursing can improve health services by creating new and innovative models of care. This treatment model must consider the fatigue factor of nurses, the needs of the millennial workforce, and how to balance the skills of new graduates and experienced care in the hospital. New technologies bring updating and flexibility in how they work. One innovative strategy is the use of virtual model nursing care(2). This literature study aims to identify the important role of telenursing in improving nursing services for patients.

\section{METHODS}

The method used in this writing is literature review which is in the form of a comprehensive summary of 10 with the following methods:

\section{Study Protocol}

The article quality guide with the PRISMA table, and the assessment guide use the Joanna Briggs Institute Guideline to determine the quality of the articles according to the theme.

\section{Article Search Strategy}

Articles were obtained by searching on 3 electronic databases, namely Scopus, Science Direct, and SAGE Journal. The keywords used according to the chosen theme are telenursingtelehealth-telemedicine- and telemonitoring.

\section{Inclusion Criteria}

The inclusion criteria for articles on telenursing are determined based on the following: journal topics on telenursing published in 2019 to 2021, directed in English, full text access, and designed both as quantitative and qualitative research with sample populations and variables related to telenursing-telehealth-telemedicine.

Guidelines in the review of these journals use the Joanna Briggs Institutute (JBI) and PRISMA, with study eligibility from its title, abstract, methodology, results and full text(6).

\section{RESULTS}

Reviews contained in 10 journals regarding telenursing from the inclusion criteria of quantitative and caulitative research designs from 3 databases electronic searches, namely Scopus, SAGE and Sciene direct, obtained the following results:

1. Article title: "The effects of Telephone-Based Telenursing on Glycated Hemoglobin Among Older Adults with Type 2 Diabetes Mellitus: A Randomized Controlled Trial."

This article started at background which conditioned poor management of Type 2 Diabetes Mellitus (DM) with acute and long-term effects that can affect various aspects of life. Patients with adequate knowledge about self-care, medications used, and lead a healthy lifestyle can 
help prevent most complications of DM. By existing technological advances, many multimedia-based teaching could be used for patient education. Telenursing is a new method of patient education and refers to the delivery of nursing care services via information technology.

Prior to the intervention, patients were given face-to-face basic education related to diabetes mellitus. This material focused on problems associated with DM, including diet, increased or decreased blood glucose levels, dietary choices, blood glucose monitoring, DM complications and prevention, DM self-care, and physical exercise recommended for elderly people with diabetes. The intervention were given for 3 months ( 12 weeks) in the control group, which was consisting of 66 eligible elderly with type DM 2 who were recruited randomly. Menawhile, this research applied the inclusion criteria for each patient which include:

-Patient should be more than 60 years old

-Patient had suffered DM fore more than 6 months

-The patient's HBA1C percentage should be more than 7\%

-Patient could access cellphone/telephone

This research found that there were no cognitive, mental, memory, speech or hearing impairments.

Of the 66 respondents, 6 of them were unable to take part in the research until the end. The comparison of $\mathrm{HbA} 1 \mathrm{C}$ levels decreased significantly in the intervention and control groups $(\mathrm{P}$ $<0.05)$. After 12 weeks of intervention, the $\mathrm{HbA1C}$ level in the intervention group was significantly lower than that in the control group $(7.20 \pm 0.82$ vs $7.90 \pm 0.74)$. However, the amount of reduction in the intervention group was significantly greater than that in the control group

The limitation of this study was that it could not control the complementary therapy of patients. After face-to-face education, the follow-up of elderly patients with type 2 diabetes mellitus could use telenursing. Telenursing could facilitate the provision of patient care and monitoring, promote active thinking among patients and their family members, teach health-promoting lifestyles and help them develop, use individual plans for the maintenance and promotion of their health. Therefore, in order to practicing inexpensive and easy-to-implement intervention, nurses could use telenursing to promote lifestyle modification, assist patients with symptom management, and improve treatment outcomes among patients with chronic disease with a lack of skill and financial status(7).

2. Article title: "The Cost-effectiveness of person-centered care provides to patients with chronic heart failure and/or chronic obstructive pulmonary disease." This article concluded that person-centered care could be provided face-to-face remotely. Once evaluated, patientcentered care by telephone was capable of having a beneficial effect. Telemedicine was tested in patients with COPD proven to improve health and rehabilitation involves a health care approach and can have a low cost effect. Patients with COPD and/or CHF would also have benefits from cost-effectiveness and improved quality of life due to directions and guidance of care given over the phone. Overall, person-centered care could provide more benefits to patients, especially low costs, but it had not been able to significantly improve the quality of physical care assistance. Thus, alternative person-centered care was more effective, resulting in higher health care costs. lower than usual care (8). 
3. Article title: "DM- calendar APP as adiabetic self-management education on adult type 2 diabetes millitus: a randomized controlled trial."

This article described the lack of communication between nurses and patients which could be caused by treatment distance and the length of time for treatment waiting in queues during treatment and control impacted the effectiveness of Diabetes Self-Management Education (DSME) management - which resulted on patients attractive to visit the hospital. The lack of opportunity for Type 2 Diabetes Mellitus (T2DM) patients to communicate about their perceived complaints caused a lack of knowledge and poor management. The use of communication technology could be a solution to this problem and as the need for interprofessional collaboration between health workers.

The DM-Calendar application is an android-based application designed by researchers as a medium for self-care education for DM sufferers living alone. The application had been provided by the researcher to be installed on the mobile respondent. This application contains three of the four 'pillars' of DM, namely blood sugar control, educational programs, nutritional therapy, and physical activity. This application system worked by reminding patients to carry out self-management programs in the form of notifications on their cellphones. The application would notify the patient six times a day: 6:00 a.m. (breakfast), 9:00 a.m. (morning snack), 12:00 p.m. (lunch), 3:00 p.m. (afternoon snack), 4:00 p.m. (physical activity/diabetic foot exercises), and 6:00 p.m. (dinner). A reminder for checking blood sugar appeared once a month from the first login. The DM calendar application was designed as a DSME medium to increase self-efficacy values, HbA1C levels, fat profiles, and insulin for type $2 \mathrm{DM}$ patients.

A total of 30 respondents from Denpasar, Bali (15 respondents for the control group and 15 respondents for the intervention group) had inclusion criteria as follows:

-Patient composted mentis

-Patient could operate android phone

-Patient with uncontrolled glycemic (HbA1C:> 7\%).

-Patient underwent the same drug therapy for at least 3 months

-Patient followed the study to completion

The results showed that the experimental group had higher self-efficacy than the control group $(\mathrm{P}<0.05)$. Likewise with the significant $\mathrm{HbA1c}$ value in the intervention group. The limitation in this research was that there is a tradition that is regularly felt called magibug (gathering and eating together). Therefore, only 30 respondents were included in this study. The hope for further research was that there will be more respondents and for a longer period of time.

The use of "telehealth" effectively increased the self-efficacy of patients with T2DM. Mobilebased application technology had been shown to improve provider communication services, escalate the accessibility for the patients, and provide the right choice to facilitate independent management processes and treatment adherence. This could be used as the reason why the use of the DM calendar application could more effectively increase the self-efficacy and activity of T2DM patients compared to conventional media (leaflets) in educational provision. A minimum of 30 days of educational program was required to keep the patient's glycemic profile under control. The use of electronic media had proven to be one of the right choices in providing continuing education more efficiently. Mobile technology and the internet had been 
widely available, accessible 24 hours a day, and could be used to promote disease management and facilitate behavior modification (9).

4. Article title: "Effect of telenursing on aging perception of Iranian older adults."

The conclusion of this article was that telenursing had been used for health care settings for the elderly who lived in communities and in their homes. The perception of aging was one of the factors of functional disability in the elderly, so this telenursing was expected to help in longdistance care efforts. According to previous research, remote care using the telephone could increased the efficiency of nurses, reduced the number of visits, and increased their working hours. Regarding the physical and functional problems of the elderly as an obstacle to being referred to a hospital or health service, telephone care benefited the elderly and allowed them to use health services without the need to move and travel a lot. This study used a quasiexperimental study with two methods, namely telephone care and face-to-face care. A total of 60 respondents (30 control groups and 30 intervention groups) with (elderly aged 60-75 years) were selected based on inclusion criteria. The research was conducted for 2 months. Patients with telephone treatment would receive calls between 08.00 - 14.00 for 10-15 minutes on certain days (10).

5. Article title: "Interaction between tele-nurses and callers with an evolving myocardial infarction: Consequences for the level of directed care."

This article explained that in patients with acute myocardial infarction (AMI), quick contact with emergency medical services was very important, but there were still many obstacles so many people turn to telehealth advisory controlled by nurse. The delivery of messages for the assessment of the patient's condition and the urgent care measures depend on how the interaction was in the call. This study used a qualitative design with 30 respondents consisting of 20 men and 10 women who were formulated from several exploratory questions including:

a. How do callers communicate symptoms and their context, and how does the nurse respond?

b. What treatment is directed and how do callers respond to the suggestions given?

The results obtained from 30 participants with an age range of 46-89 years found a communication structure that was divided into 3 phases, namely opening, orienting, and ending. The main findings revealed one overall category namely 'movement towards targeted care levels' and four categories: 'distinct', 'reasoning', 'indecisive' and 'irrational' interactions between nurse and caller in the care setting of patients with acute myocardial infarction. When Swedish Healthcare Direct was selected as First Medical Contact, the four findings were as follows:

a. Distinct interaction $\rightarrow \mathrm{A}$ brief and forth directed interaction

b. Reasoning interaction $\rightarrow$ An interaction which characterized by collaboration that was reflected in achieving mutual understanding. This interaction was usually found in the orientation phase. The caller described the symptoms and the nurse collects information about the situation and could capture a broad description of the symptoms.

c. Indecisive interaction $\rightarrow$ An interaction which characterized by a vague and unclear dialogue, in terms of understanding the accuracy of communication.

d. Irrational interaction $\rightarrow$ An interaction which characterized by how the nurse and caller were connected incoherently.

When the interaction between the nurse and the caller was unclear, there was a high risk that the caller would not follow the advice given in the call. Nurses also had difficulty identifying symptoms that appeared to require acute care. Summarized calls on a nonproductive basis could also led to failure in patient care (11). 
6. Article title: "Video consultation as nursing practice during early in-home care for premature infants and families viewed from the families 'homes', telenursing on the care of premature babies using video."

As the beginning, this article explained premature babies need treatment because organ immaturity occured as a result of birth before 37 weeks' gestation. Families with premature babies were offered to do home care from an early age. In Denmark, early home care programs had become standard care. The use of video consultations had been carried out in the care of premature babies in recent years. This study examined how the communication between nurses and families in a video consultation on a home baby care program from an early age. This qualitative study based on focused observation supported by video consultations and audio recordings. Five participants were involved in this study and nurses with 2.5-20 years experience in the neonatal field aged 32-63 years. The findings obtained several themes:

a. Setting the scene: This theme covered the context in which video consultations were conducted in the family home on an early home care program.

$\checkmark$ The location of the consultation category: Mother was at home with father and/or baby's older sibling. They had already planned where the consultation would take place.

$\checkmark$ Pausing daily life category

b. Weight as a point of reference: This theme led questions and discussions around the baby's weight, and talked less about the well-being of the family and the baby.

$\checkmark$ The infant's weight category

$\checkmark \quad$ Short and closed questions category

$\checkmark$ Acquaintance category

c. The pros and cons of technology: This theme conceived some interference when communicating may occured which creating divisions in communication.

$\checkmark \quad$ Keeping the consultation on track category

$\checkmark$ Confidence with the technology category: This category directed to the nurse's lack of confidence in using video. Consequently, their findings emphasized that the use of video consulting requires training in video communication and video usage beliefs.

In the future, the related research could combine participant observation with communication during consultations. In this research, video consultations provided easy and enjoyable access to home care for families. However, the videos' potention, which were supposed to gather a lot of information about babies or families, had used only to a limited extent. Video consultations were disrupted, making it difficult for nurses to keep consultations on track (12).

7. Article title: "Data-work for personalized care: Examining nurses' practices in remote monitoring of chronic patients."

This study described a new paradigm of the care for chronic disease patients with a remote monitoring model. The use of remote monitoring systems and IT devices allowed healthcare professionals to capture patient data sets and monitor them from a distance. Remote patient monitoring systems had grown in popularity in recent years. This was because of its potential to improve medical services by providing more affordable, timely and accessible care. The use of telemonitoring systems had also been shown to increase the frequency of interactions between patients and healthcare providers, and a number of systematic reviews of research studies had shown that telemonitoring benefited patients by promoting their involvement in care practices.

The research, which began in 2016 to 2017, used an application called MyDignio, a remote monitoring for patients with chronic diseases, where patients could have full independent 
access to the results and could communicate with health services. The data were collected by interviewing and observing the respondents.

There were three main data that supported personalization: preparatory work, continuous adjustment, and patient assistance in establishing routines to generate relevant data with the right questions.

a. Preparatory work: identifying core health problems

b. Continuous adjustment: understanding how to monitor what is relevant

c. Refining the question.

This remote care of data played a more central role than in traditional settings. Nurses had a new role in telemonitoring and this was changing the way things work based on specific knowledge and skills. This technology relied on specially trained nurses who were dedicated to reading and interpreting monitoring data(13).

8. Article title: "Development of the Teaching Interpersonal Skills for Telehealth Checklist" This article described distance care education methods, with the aim of developing a valid and reliable model to make it easier for educators and managers to evaluate interpersonal skills in care. Researchers used a multi-stage iterative approach to develop and test the validity and reliability of the Teaching Interpersonal Skills in Telehealth checklist (TIPS-TC). Through the development and testing of TIPS-TCs, interpersonal skills were identified that could be used to assess CMC during video consultations with telehealth. Checklist testing could help evaluate student and clinician competencies. This evaluation could be used to assess and improve a clinician's interpersonal and communication skills. The ability to assess interpersonal skills in telehealth could assist clinical education efforts to develop effective communication between doctors and patients. After further study, the use of the TIPS-TC could inform evidence-based curricula of educational interventions for physicians and students across a wide range of disciplines for the development of interpersonal skills with telehealth. One of the expected capacity development programs was the telehealth program to be easy and commonplace. Telehealth benefits could also increase interpersonal skills learning activities to have a positive impact on students, professionals, and care outcomes (14).

9. Article title: "Feasibility, acceptability, and preliminary impact of telemedicineadministered cognitive behavioral therapy for adherence and depression among African American women living with HIV in the rural South." This article explained the benefits of telemedicine to help people with HIV (PLWHA) avoid major depression which is at risk of aggravating the condition. People living with HIV (PLWHA) were indirectly burdened by depression. People who were depressed are associated with an increased risk of death. Gender differences in the incidence of depression indicate higher rates for women living with HIV than for men with HIV and for women who were not affected by HIV. Telemedicine had been found to be helpful in overcoming barriers to health care, particularly in rural areas where access to special care such as psychiatry was very limited and often non-existent. Interventions distributed via telemedicine could help overcome barriers to access to mental health care while providing expertise in their fields.

Random data collection and women with HIV would receive intervention or supertive psychotherapy administered via video conferencing. The mix method was used to collect data from recipients of the intervention at the time of using video via letemedicine in counseling sessions. Participants who took part in this study were 22 people who would receive direct intervention via telemedicine with psychiatry for 1 hour every week for 10-12 weeks (3). 
10. Article title: "Telephone follow-up of the elderly after cataract surgery describes telenursing in post-cataract surgery patients." In this article, it had been known that cataracts could be treated with surgery which was expected to have a postoperative recovery time of about 3-7 days thereafter. However, the postoperative complication rate could reach $14.7 \%$ whose main factors include increased intraocular pressure, corneal edema, posterior capsule opacity, inflammatory processes, and endophthalmitis. This requires postoperative follow-up strategies such as patient monitoring to detect problems early through virtual consultation.

This research was conducted randomly and there was a control group. The inclusion criteria for each respondent was a person aged 60 years or over, would undergo cataract surgery, had cell phones, and could operate them to communicate with nurses. Respondents who participated in this study were 95 patients ( 48 for the intervention group and 47 patients for the control group). The effect of telephone follow-up intervention performed by nurses resulted in a significant difference in the surgical recovery of patients in the experimental group compared with patients in conventional treatment. The control group showed a higher incidence of delayed surgical recovery, with an 8.5 times greater likelihood of presenting complications $(\mathrm{OR}=0.118 ; \mathrm{CI}=1.02,10.23)$ in the second evaluation (performed on the seventh postoperative day). These findings suggested that telephone monitoring could greatly protect against postoperative recovery delays (15)

\section{DISCUSSION}

Telenursing was developed through research and applied in various conditions, both cases of acute disease, chronic disease, surgery, and cases in the elderly or toddlers, as described in the 10 journals summarized in the results above. The conclusion of the ten journals on average describes the education and direction of various conditions conveyed by nurses to clients and/or families.

Patients with Type $2 \mathrm{DM}$ in the elderly need supervision so that their compliance with medication can be routinely monitored. A person diagnosed with COPD with long-term home care can be serviced over the phone to reduce higher costs. Patients who have cardivascular disorders, especially acute myocardial infarction (AMI), by using telenursing service, will experience an effective assessments and emergency situations protocol because it will speed up nurses' response for patients to get further treatment. Another research, furthermore, mentioned that the use of telenursing in the form of nursing home residents (NHR) can reduce the number of elderly people in nursing homes who experience an emergency having to go to the hospital and/or reduce the cost of unnecessary laboratory examinations(16).

Premature babies than babies with normal births were more fragile and sensitive to the environment, therefore the need for vigilance in care (17). Premature babies in home care could be treated by telenursing in order to properly provide independent care for the mother and baby's family and can grow the baby healthily according to the development of a child concept. Telemonitoring for patients with chronic diseases is treated with the help of equipment to monitor conditions remotely, which is it will not reduce the frequency of patient monitoring even in different places. The telehealth checklist (TIPS-TC) was developed for educators or clinical instructors (CI) to provide monitoring skills for students who are carrying out nursing care appropriately without having to face to face directly. Monitoring carried out in nursing is a key task for a nurse and in this era advanced technological support is reqirued, such as the use of Self-Monitoring Analysis and Reporting Technology (S.M.A.R.T.), which designed to monitor and analyze and provide information on various kinds of problems. This technology also makes it easy for remote care (5). 
Telenursing is also used for PLWHA survivors as an effort to reach outreach care to avoid depression as a common complication that occurs in people with HIV. In patients undergoing cataract surgery, telenursing is also effective to avoid complications after surgery. The use of video media in telenursing is also important to avoid the subjectivity factor of a sign in a patient's physical examination that is needed for data analysis and problems that occur, as in this study related to telenursing using video on palliative care proving interactive video is used to pay attention to progress or decline. physical patient. This is done in order to ease patients to might not come to the hospital. Thus, it reduces the time and physical activity to come to health facilities(18).

Telenursing-telemonitoring-telehealth is more on the aspect of intervention in the form of education and emotional support. A patient and/or family will really need knowledge of how to care for themselves or their family who are experiencing illness without having to come to the hospital. Thus, with its characteristic, telenursing will overcome their problem. This is in line with other studies which illustrate that providing health education through telenursing was effective in improving the quality of life of burn patients during the rehabilitation phase (19).

Another description of telenursing in education is that the divergent modes of relational experience are autonomy, dependence, domination and neglect. This illustration is possible because the previous research argued that interpersonal connectivity work involved an act of balancing a duality or two aspects(20). Physical nursing skills will indeed decrease in quality if it is practiced virtually, but several aspects of tele-informed, tele-communication and virtual care culture fluency can be applied optimally (21).

Telenursing with the support of developing information technology is a nursing paradigm that must be mastered by nurses in the 21 st century, and will be left behind if they do not follow it, as the demands of various patients have been escalated and with the principle of being more efficient in terms of time and cost. Thus, nurses must can fulfill this with longdistance care efforts. This is in line with the case of Covid -19 pandemic where there are many limiting factors for continuous interaction. Telemedicine with the support of high-capable technology such as 5G, then, would help care for remote patients with good communication quality and clearly, although the users of this service would incured of higher connection fees (4).

Telenursing is also applied in various ways, one of which is for the needs of psychiatric care. In general, primary psychiatric health services do not have sufficient time, so telepschiatriy is used. Based on previous study, it can be concluded that there were consistency of the diagnostic accuracy and satisfaction of patients and service providers, as well as decreased psychiatric hospitalizations, and the effectiveness of treatment time and costs, as the application of telenursing (22). This broad view of various aspects of the world of nursing in this millennial century is a reflection as well as a challenge to continue to be developed in nursing specialties to use digitalized technology and information technology. Telenursing findings will be fruitful in improving nursing services in particular and or health services in general. Telehealth nursing practice utilized technology-based communication to provide remote patient nursing services such as assessment, providing educational interventions, monitoring and evaluating patient care (23). Another study showed that nurses were satisfied with facilitating patient data on a primary PC or personal tablet to be able to monitor intravenous fluids as well as some drugs that could be monitored from outside the patient's room (24). This technology support is part of efforts to be effective in accurate monitoring.

Nursing in the 21 st century answers the challenge through telenursing with other subtele which support each other. The nurse as a subject has a comprehensive role, including the role of developing theories and models in nursing. The philosophical and contextual issues in nursing theory in the 21 st century have also been developed in the theory of caring models in 
technology-based care(25). Nurses in carrying out nursing care will have a lot of progress, innovation, and effectiveness in responding to social demands for a paradigm shift in nursing care that leads to the need for satisfaction for family and community patients (26).

\section{CONCLUSION}

The 21st century global nursing paradigm was developed with the help of technology to meet the needs of services that have distance, physical and cost limitations. Telenursing is a solution to answer the challenges of the demands of efficient and quality health services. The summary of 10 articles shows that there are significant benefits to the use of technology to support health services. Nursing has many ways to facilitate the patient's need for nursing care and/or in managing management and conducting distance education that is more efficient and can reduce costs. Telehealth, telemonitoring, telepsychiatry are part of telenursing which can be done as the remote care for patients who need fast help such as the IMA case to get directions for handling assistance and monitoring quickly and accurately; monitoring services for sufferers of chronic disease; monitoring of medication adherence for type 2 diabetes mellitus and COPD patients; care education for mothers and families who have premature babies; remote care for post-cataract surgery patients; and prevention of major depression in people living with HIV/AIDS by utilizing digital technology for teleconfigured videos. This is a manifestation of the nursing paradigm in the 21 st century that uses technology to provide nursing care.

\section{REFERRENCE}

1. Ortiz MR. The Patient-Centered Health Neighborhood. Nurs Sci Q. 2020;33(4):353-7.

2. Cloyd B, Thompson J. Virtual Care Nursing:: The Wave of the Future. Nurse Lead [Internet]. 2020;18(2):147-50. Available from: https://doi.org/10.1016/j.mnl.2019.12.006

3. Nejadshafiee M, Bahaadinbeigy K, Kazemi M, Nekoei-Moghadam M. Telenursing in Incidents and Disasters: A Systematic Review of the Literature. J Emerg Nurs. 2020;46(5):611-22.

4. Siriwardhana Y, Gür G, Ylianttila M, Liyanage $\mathrm{M}$. The role of $5 \mathrm{G}$ for digital healthcare against COVID-19 pandemic: Opportunities and challenges. ICT Express [Internet]. 2020;(xxxx). Available from: https://doi.org/10.1016/j.icte.2020.10.002

5. Locsin RC, Soriano GP, Juntasopeepun P, Kunaviktikul W, Evangelista LS. Social transformation and social isolation of older adults: Digital technologies, nursing, healthcare. Collegian [Internet]. 2021; $(x x x x)$ Available from: https://doi.org/10.1016/j.colegn.2021.01.005

6. Literature_Systematic_Review_pada_Pendid (1).

7. Esmaeilpour-BandBoni M, Gholami-Shilsar F, Khanaki K. The Effects of TelephoneBased Telenursing on Glycated Hemoglobin Among Older Adults With Type 2 Diabetes Mellitus: A Randomized Controlled Trial. J Nurse Pract [Internet]. 2020;(xxxx). Available from: https://doi.org/10.1016/j.nurpra.2020.09.015

8. Pirhonen L, Gyllensten H, Olofsson EH, Fors A, Ali L, Ekman I, et al. The costeffectiveness of person-centred care provided to patients with chronic heart failure and/or chronic obstructive pulmonary disease. Heal Policy OPEN [Internet]. 2020;1:100005. Available from: https://doi.org/10.1016/j.hpopen.2020.100005

9. Kusnanto, Widyanata KAJ, Suprajitno, Arifin H. DM-calendar app as a diabetes selfmanagement education on adult type 2 diabetes mellitus: a randomized controlled trial. J Diabetes Metab Disord. 2019;18(2):557-63. 
10. Sadeghmoghadam L, Ahmadi Babadi S, Delshad Noghabi A, Nazari S, Farhadi A. Effect of telenursing on aging perception of Iranian older adults. Educ Gerontol [Internet]. 2019;45(7):476-82. Available from: https://doi.org/10.1080/03601277.2019.1657263

11. Ericsson M, Ängerud KH, Brännström M, Lawesson SS, Strömberg A, Thylén I. Interaction between tele-nurses and callers with an evolving myocardial infarction: Consequences for level of directed care. Eur J Cardiovasc Nurs. 2019;18(7):545-53.

12. Hägi-Pedersen MB, Kronborg H, Norlyk A. Video consultation as nursing practice during early in-home care for premature infants and families viewed from the families' homes'. Nurs Open. 2020;(October):1-9.

13. Grisot M, Moltubakk Kempton A, Hagen L, Aanestad M. Data-work for personalized care: Examining nurses' practices in remote monitoring of chronic patients. Health Informatics J. 2019;25(3):608-16.

14. Henry BW, Billingsly D, Block DE, Ehrmann J. Development of the Teaching Interpersonal Skills for Telehealth Checklist. Eval Health Prof. 2021;016327872199283.

15. Machado TMD, Santana RF, Vaqueiro RD, Santos CTB dos, Alfradique de Souza P. Telephone follow-up of the elderly after cataract surgery. $\mathrm{Br} \mathrm{J}$ Vis Impair. 2020;38(2):184-95.

16. Brickman KR, Silvestri JA. The emergency care model: A new paradigm for skilled nursing facilities. Geriatr Nurs (Minneap) [Internet]. 2020;41(3):242-7. Available from: https://doi.org/10.1016/j.gerinurse.2019.10.004

17. Jiang X. Analysis on the Main Points of Nursing Premature Infants 2 . Pay attention to the adjustment of light 4 . Grading according to specific de- livery weeks 3 . Reduce the stimulation of envi- ronmental noise care. 2020;9(4):71-4.

18. Jess M, Timm H, Dieperink KB. Video consultations in palliative care: A systematic integrative review. Palliat Med. 2019;33(8):942-58.

19. Rezaei M, Jalali R, Heydarikhayat N, Salari N. Effect of Telenursing and Face-to-Face Training Techniques on Quality of Life in Burn Patients: A Clinical Trial. Arch Phys Med Rehabil [Internet]. 2020;101(4):667-73. Available from: https://www.sciencedirect.com/science/article/pii/S0003999319314443

20. Hafermalz E, Riemer K. Interpersonal Connectivity Work: Being there with and for geographically distant others. Organ Stud. 2020;41(12):1627-48.

21. Rambur B, Palumbo MV, Nurkanovic M. Prevalence of Telehealth in Nursing: Implications for Regulation and Education in the Era of Value-Based Care. Policy, Polit Nurs Pract. 2019;20(2):64-73.

22. Finley BA, Shea KD, Maixner R, Slebodnik M. Advanced Practice Registered Nurses Using Synchronous Telepsychiatry: An Integrative Systematic Review. J Am Psychiatr Nurses Assoc. 2020;

23. Watkins S, Neubrander J. Primary-care registered nurse telehealth policy implications. J Telemed Telecare. 2020;

24. Feldenzer K, Rosenzweig M, Soodalter JA, Schenker Y. Nurses' perspectives on the personal and professional impact of providing nurse-led primary palliative care in outpatient oncology settings. Int J Palliat Nurs. 2019;25(1):30-7.

25. Sataloff RT, Johns MM, Kost KM. No 主観的健康感を中心とした在宅高齢者にお ける 健康関連指標に関する共分散構造分析Title. :8-11.

26. Oh EG, Jang Y, Park J, Lee H, Kim H, Min A, et al. Development of Strategic Plans for Advancing Nursing in Korea. Asian Nurs Res (Korean Soc Nurs Sci) [Internet]. 2019;13(2):115-21. Available from: https://doi.org/10.1016/j.anr.2019.02.003 